\title{
Weak negative associations between avian influenza virus infection and movement behaviour in a key host species, the mallard Anas platyrhynchos
}

\author{
Jacintha G. B. van Dijk, Erik Kleyheeg, Merel B. Soons, Bart A. Nolet, Ron A. M. Fouchier and \\ Marcel Klaassen
}

J. G. B. van Dijk (jacintha.vandijk@gmail.com), M. B. Soons, B. A. Nolet and M. Klaassen, Dept of Animal Ecology, Netherlands Inst. of Ecology (NIOO-KNAW), Droevendaalsesteeg 10, NL-6708 PB Wageningen, the Netherlands. - MBS and E. Kleyheeg, Inst. of Environmental Biology, Utrecht Univ., Padualaan 8, NL-3584 CH Utrecht, the Netherlands. MK also at: Centre for Integrative Ecology, School of Life and Environmental Sciences, Deakin Univ., Locked Bag 20000, Geelong, VIC 3220, Australia. - R. A.M. Fouchier, Dept of Viroscience, Erasmus MC, PO Box 2040, NL-3000 CA Rotterdam, the Netherlands.

\begin{abstract}
Animal movements may contribute to the spread of pathogens. In the case of avian influenza virus, [migratory] birds have been suggested to play a role in the spread of some highly pathogenic strains (e.g. H5N1, H5N8), as well as their low pathogenic precursors which circulate naturally in wild birds. For a better understanding of the emergence and spread of both highly pathogenic (HPAIV) and low pathogenic avian influenza virus (LPAIV), the potential effects of LPAIVs on bird movement need to be evaluated. In a key host species, the mallard Anas platyrhynchos, we tested whether LPAIV infection status affected daily local $(<100 \mathrm{~m})$ and regional $(>100 \mathrm{~m})$ movements by comparing movement behaviour 1) within individuals (captured and sampled at two time points) and 2) between individuals (captured and sampled at one time point). We fitted free-living adult males with GPS loggers throughout the autumn LPAIV infection peak, and sampled them for LPAIV infection at logger deployment and at logger removal on recapture. Within individuals, we found no association between LPAIV infection and daily local and regional movements. Among individuals, daily regional movements of LPAIV infected mallards in the last days of tracking were lower than those of non-infected birds. Moreover, these regional movements of LPAIV infected birds were additionally reduced by poor weather conditions (i.e. increased wind and/or precipitation and lower temperatures). Local movements of LPAIV infected birds in the first days of tracking were higher when temperature decreased. Our study thus demonstrates that bird-assisted dispersal rate of LPAIV may be lower on a regional scale than expected on the basis of the movement behaviour of non-infected birds. Our study underlines the importance of understanding the impact of pathogen infection on host movement in order to assess its potential role in the emergence and spread of infectious diseases.
\end{abstract}

Animal movements may assist in the spread of pathogens (Altizer et al. 2011). However, the impact of the pathogens on host movement behaviour is of critical importance for pathogen spread. Some studies show that pathogens may reduce their host's capacity for locomotion (Bradley and Altizer 2005, Fellous et al. 2011). Pathogens may also reduce a host's food intake and therewith body condition (Delahay et al. 1995), which may in turn reduce the time spent in locomotion (Yorinks and Atkinson 2000). Hence, when studying the role of animal movement in the transmission of pathogens, it is vital to include potential effects of infection on the host's locomotion ability, since this may affect the local and global spread of pathogens.

One important pathogen that is suggested to be spread by bird movements is avian influenza virus (AIV). The low pathogenic phenotype of AIV (LPAIV) circulates globally in waterfowl and shorebirds (orders Anseriformes and Charadriiformes), which are considered the natural LPAIV reservoirs (Webster et al. 1992). Waterfowl are thought to transport LPAIV asymptomatically over large distances, since infection causes only mild disease (Kuiken 2013). Studies in naturally infected waterfowl showed minor differences in body mass between LPAIV infected and non-infected birds, with weak negative associations between infection and immune status (Latorre-Margalef et al. 2009, Kleijn et al. 2010, van Dijk et al. 2015). However, few studies have investigated the potential effects of LPAIV infection on waterfowl movements. A study in migratory Bewick's swans Cygnus columbianus bewickii fitted with GPS neck-collars showed that LPAIV-infected birds were feeding at reduced rates and left one month later for spring migration (van Gils et al. 2007). Conversely, a follow-up study in the same species using a much larger sample size yielded inconclusive results (Hoye 2011), and a study using banding recoveries of mallard ducks Anas platyrhynchos found no effects of LPAIV infection on migration speed and distance (Latorre-Margalef et al. 2009). Besides LPAIV, waterfowl are also suggested to be involved in the spread of highly pathogenic phenotypes 
of AIV (HPAIV) (Kilpatrick et al. 2006). LPAIV subtypes H5 and $\mathrm{H} 7$ may mutate towards HPAIV after spill-over into poultry, which is lethal for poultry and may cause illness and occasional deaths in humans and wild birds (Alexander 2007). Several studies have correlated migration routes of wild waterfowl to HPAIV outbreak events (Prosser et al. 2009, Takekawa et al. 2013). However, there is no conclusive evidence that waterfowl are indeed transporting HPAIV around the globe, even though they are occasionally infected (Hesterberg et al. 2009).

A better understanding of the potential effects of LPAIV infection on waterfowl movements is vital for developing predictive models on the emergence and spread of both LPAIV and HPAIV, since previous outbreaks showed that these infectious diseases may form a serious threat to animal and public health, as well as the economy. For instance, LPAIV H7N9 that emerged in February 2013 in poultry in China has a high fatality rate in humans (more than 100 deaths; World Health Organization 2014a), despite being low pathogenic to poultry and other birds (Kreijtz et al. 2013). Since its emergence in 1996 in Asia, HPAIV H5N1 has led to the culling of hundreds of millions of poultry, and also resulted in almost 400 human deaths (World Health Organization 2014b). Other HPAIV strains of concern have emerged since, such as H5N8 in South Korea (Kim et al. 2014, Lee et al. 2014) that has just recently also been discovered in poultry and wild ducks in Europe.

In addition to migratory movements, regional waterfowl movements are likely to play a role in the transmission of LPAIV to other wild birds and the potential introduction of this virus into poultry. LPAIV is transmitted primarily by the faecal-oral route, with virus particles in faeces shed into the surface water and being ingested by waterfowl with water (Webster et al. 1992). Since LPAIV infections last approximately one week in wild waterfowl (Latorre-Margalef et al. 2009), this provides ample opportunities for infected birds to spread the virus in the surrounding area when undertaking daily regional flights, putting other birds, including poultry, at risk of infection.

The aim of our study was to investigate associations between LPAIV infection and local and regional movements of wild waterfowl. Throughout the autumn LPAIV infection peak, we recorded daily movement patterns of infected and non-infected mallards, a key LPAIV host species, at a high temporal and spatial resolution using GPS loggers. We examined associations between LPAIV infection status and movement metrics in two ways: firstly by testing whether within-individual changes in infection status altered movement behaviour in birds captured at two points in time (in infected and non-infected state, or vice versa), and secondly by comparing infected and non-infected individuals. If LPAIV infection is negatively associated with mallard movement behaviour, this will likely reduce the local and regional spread of this infectious disease.

\section{Methods}

\section{Study species and site}

Mallards are one of the most common and numerous waterfowl species in the world (estimated population
19 million individuals; Delany and Scott 2006). Together with other dabbling ducks of the Anas genus, mallards are frequently infected with LPAIV and harbour most virus subtypes found in birds to date (Olsen et al. 2006). In the northern hemisphere, a major LPAIV infection peak in mallards occurs in autumn, with a decrease in virus prevalence in winter, after which infection rates increase in spring, resulting in a minor infection peak in summer (Latorre-Margalef et al. 2014, van Dijk et al. 2014). The autumn peak is likely driven by aggregation of birds, importantly involving susceptible migrants, while the summer peak coincides with the entrance of immunologically naïve juveniles into the population (van Dijk et al. 2014).

Our study site was located in the Alblasserwaard $\left(51^{\circ} 52^{\prime} 38^{\prime \prime} \mathrm{N}, 4^{\circ} 43^{\prime} 26^{\prime \prime} \mathrm{E}\right.$; Fig. 1), the Netherlands. Mallards in the Netherlands belong to the northwestern European population, which is partially migratory, consisting of migratory and resident birds. Migratory mallards breed in northern Europe (i.e. Finland, Sweden, the Baltic, northwest Russia) and migrate in autumn to winter from Denmark to France and Britain. On the wintering grounds, migratory mallards congregate with residents that breed in western Europe (e.g. the Netherlands; Scott and Rose 1996). At our study site, resident mallards are present throughout the year, while migratory mallards arrive in autumn to winter.

\section{Sampling}

During the peak of the LPAIV infection period (autumn and winter) in 2012, 74 free-living mallards were captured and recaptured using a duck decoy, consisting of five swim-in traps connected to a large pond. Wheat grain was provided during the day to attract mallards, which was often consumed immediately. Mallards were (re)captured throughout the end of summer, autumn and winter (August-January). A pilot study at this location in spring 2012, in which 20 mallards were equipped with GPS loggers, revealed that the duck decoy was used as a roosting site during the day, with morning and evening flights to feed in the surrounding area (Kleyheeg unpubl.). These results are consistent with studies that recorded regional movements of mallards on the wintering grounds that were characterized by daily flights, mostly at sunset and sunrise, between roosts and foraging sites with distances of up to tens of kilometres (Kleyheeg unpubl., Sauter et al. 2012).

For each captured bird, plumage characteristics were used to determine age and sex. Previously, no difference was observed in LPAIV prevalence between male and female mallards (Munster et al. 2007). Only adult male mallards $(>1$ year old) were used in order to avoid age and sex-related variance in movement patterns. Another reason for using only males in our study was the relatively high chance of capturing pairs (most pairs are formed by late October; Cramp and Simmons 1977); it was expected that pairs would have similar movement patterns. Each bird was fitted with a unique identifying ring. Three biometric measurements were taken to assess bird size: tarsus length (nearest $0.01 \mathrm{~mm}$ ), head + bill length (nearest $0.1 \mathrm{~mm}$ ) and wing length (maximum wing chord, nearest $1 \mathrm{~mm}$ ). Body mass was measured with a digital balance (nearest $1 \mathrm{~g}$ ) to represent each bird's energetic condition after correction of body 


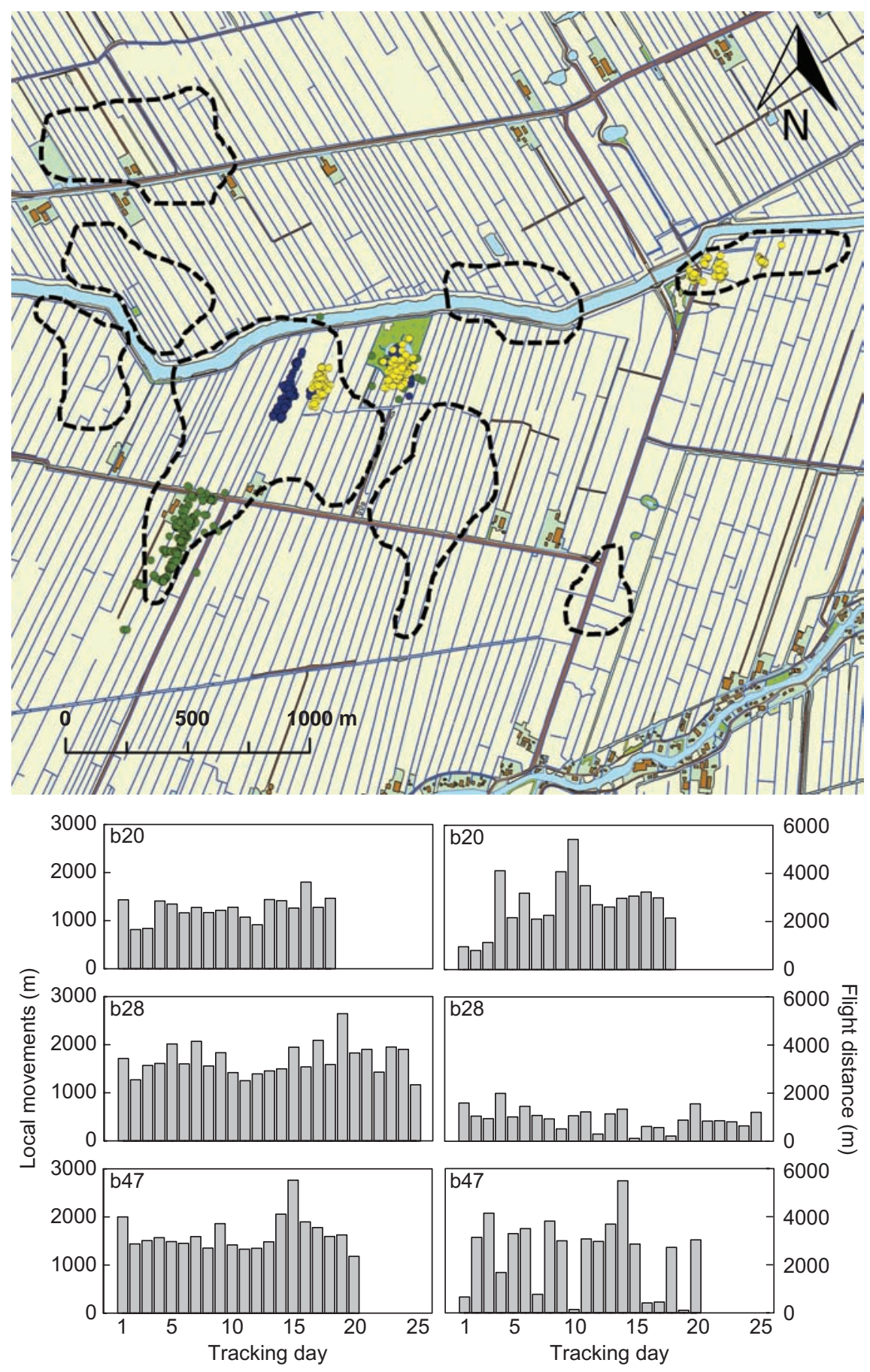

Figure 1. Map of the study area with the GPS positions of three (randomly chosen) individual mallards: b20 (green dots), b28 (blue dots) and b47 (yellow dots). The map depicts the roosting site (i.e. duck decoy) in the centre, and the main foraging areas are marked with dotted lines (foraging areas were visited by all GPS logged mallards). The straight narrow lines perpendicular to the wide canal represent ditches. For each individual mallard, the cumulative local movements (left panels) and flight distance (right panels) per tracking day are shown. If the distance between two GPS positions at the roost or in a foraging area were $<100 \mathrm{~m}$, movements were defined as local movements. If the distance between two GPS locations were $>100 \mathrm{~m}$, for instance between the roost and a foraging area or between two foraging areas, the movements were defined as flight (i.e. regional movements).

size. Blood samples $(0.5-1.0 \mathrm{ml},<2 \%$ of the circulating blood volume) were collected from the brachial vein for detection of antibodies to AIV. Blood was allowed to clot for approximately $6 \mathrm{~h}$ before centrifugation to separate serum from red blood cells (Hoye 2012), and was stored at $-20^{\circ} \mathrm{C}$ until analysis. Sterile cotton applicators were used to swab both the cloaca and the oropharynx for detection of current LPAIV infection. Experimental infection studies in mallards show that LPAIV replicates in the intestinal tract and, in lower titres, in the respiratory tract (Kida et al. 1980). Swabs were stored individually in transport medium (Hank's balanced salt solution with supplements; Munster et al. 
2007), preserved at $4^{\circ} \mathrm{C}$ and transported to Erasmus MC for analysis. Cloacal and oropharyngeal samples were also collected from birds when recaptured to remove the GPS logger.

\section{GPS loggers}

Each bird was equipped with a GPS logger $(45 \times 25 \times$ $15 \mathrm{~mm}$ ), fitted to the bird as a backpack with a teflon harness (Roshier and Asmus 2009). The minimal costs of these loggers enabled us to maximize our sample size. Birds were recaptured to read-out the data. The time between logger deployment and recovery varied between 12 and 31 days. The all-up weight of GPS logger and harness was approximately $30 \mathrm{~g}$, which is around $3 \%$ of the body mass of male mallards. The GPS loggers were programmed to record a bird's position at 15 min intervals resulting in 96 fixes per day. Based on these $15 \mathrm{~min}$ intervals, battery life was expected to be at least 14 days. We measured spatial accuracy of the GPS loggers at three locations, in which three loggers per location were fixed to a pole. Based on 24081 positions and after excluding obvious misreadings exceeding $100 \mathrm{~m}(0.6 \%$ of the positions), average logger error was $10 \mathrm{~m}$.

\section{Movement parameters}

Logged GPS positions were used to calculate movements of individual birds for each recorded $24 \mathrm{~h}$ period, measured from 12:00 noon until 12:00 noon the next day. This period was chosen in order to fully cover the period that birds were foraging at night. Noon tends to be a period of inactivity (i.e. rest). Only days that covered a full $24 \mathrm{~h}$ period were used in the data analyses. Moreover, to avoid potential handling effects on movement patterns, GPS locations recorded until noon on the day following GPS deployment were excluded.

Five movement parameters were calculated: 1) daily local movements $\left.\left(\mathrm{m} \mathrm{d}^{-1}\right), 2\right)$ daily number of flights $\left.\left(\mathrm{d}^{-1}\right), 3\right)$ daily flight distance $\left.\left(\mathrm{m} \mathrm{d}^{-1}\right), 4\right)$ daily home range $\left(\mathrm{km}^{2} \mathrm{~d}^{-1}\right)$ and 5) daily time away from the roost (i.e. the duck decoy; $\min d^{-1}$ ). Before parameter estimation, we first removed clearly erroneous GPS fixes. These were identified as sudden large apparent displacements (i.e. $>100 \mathrm{~m}$ ) followed by an immediate return to the original position. Local movements were considered to be within-patch movements (i.e. within the roost or foraging site) of less than $100 \mathrm{~m}$ between two positions (readings 15 min apart; Fig. 1). Regional movements were considered to be movements that exceeded 100 $\mathrm{m}$ between two positions, which are mainly flights, but may occasionally involve swimming behaviour (Fig. 1). Since foraging areas were relatively close to the roosting site (mean \pm SE: $388 \pm 32 \mathrm{~m}$ ), we selected an arbitrary threshold of 100 $\mathrm{m}$ in order to quantify displacements between these sites as regional movements. We defined 'daily local movements' as the cumulative distance of local movements covered within $24 \mathrm{~h}$. Per $24 \mathrm{~h}$ recording period, the 'daily number of flights' was the total number of such $>100 \mathrm{~m}$ displacements, while 'daily flight distance' was defined as the total accumulated distance birds covered regionally during these events. We used the term 'flight', since most of the $>100 \mathrm{~m}$ displacements were flights (GPS positions on land) instead of swimming behaviour (GPS positions in water). 'Daily home range' was calculated over the same $24 \mathrm{~h}$ recording period and based on the concept of utilization distribution (van Winkle 1975), in which an animal's range is described by a bivariate probability density function of location over a period of time (i.e. the probability of finding an animal in a defined area within its home range with a confidence region set at 90\%). A bird's utilization distribution was calculated with the kernelUD function of the $\mathrm{R}$ package adehabitatHR (Calenge 2006). 'Daily time away from the roost' was defined as the period that birds were $>50 \mathrm{~m}$ from the duck decoy for at least two consecutive positions over the $24 \mathrm{~h}$ recording period.

\section{Weather conditions}

We included weather conditions in our study to account for potential weather effects on mallard movements (Sauter et al. 2012). We used hourly measurements of average wind speed $\left(\mathrm{m} \mathrm{s}^{-1}\right)$ and temperature $\left({ }^{\circ} \mathrm{C}\right)$, and sums of precipitation $(\mathrm{mm})$ collected by the Royal Netherlands Meteorological Institute (KNMI) at Cabauw ( $\left.51^{\circ} 57^{\prime} 55^{\prime \prime} \mathrm{N}, 4^{\circ} 53^{\prime} 52^{\prime \prime} \mathrm{E}\right)$, situated $16 \mathrm{~km}$ from the duck decoy. For each $24 \mathrm{~h}$ period, we calculated mean wind speed, mean temperature and total precipitation to serve as covariates in the analyses. For mean monthly averages of these weather parameters, see Supplementary material Appendix 1 Fig. A1.

\section{Virus and antibody detection}

Detection of viral presence was used as the main indicator of LPAIV infection status, whereas AIV-specific antibody presence was included as a covariate in those analyses for which past exposure was considered relevant.

To detect influenza A virus, cloacal and oropharyngeal swabs were taken. For full details on RNA isolation and virus detection, see Munster et al. (2007). In short, a MagNA Pure LC Total Nucleic Acid Isolation Kit with a MagNA Pure LC System was used to isolate RNA. Influenza A virus was detected using a generic real-time reverse transcriptase PCR assay targeting the matrix gene. Birds were considered LPAIV positive when either cloacal or oropharyngeal samples were positive.

Serum AIV-specific antibodies (i.e. antibodies that bind to the highly conserved nucleoprotein of AIV) were measured using a commercially available blocking enzymelinked immunosorbent assay (bELISA MultiS-Screen Avian Influenza Virus Antibody Test Kit) following manufacturer's instructions. Samples were tested in duplicate, with two positive and negative controls per plate, and the absorbance was measured at $620 \mathrm{~nm}$ using an infinite M200 plate reader. Samples were considered AIV antibody positive, if the signal-to-noise ratio (i.e. the absorbance of the samples divided by the mean absorbance of the negative control) was $<0.5$.

\section{Data analysis}

To test whether LPAIV infection was associated with movement patterns within and among birds, we used the recordings of the first three days after GPS logger deployment and the last three days before logger removal. It was assumed that birds that were infected with LPAIV on the day of logger deployment (i.e. day 0 ) were also infected during the 
following three days (i.e. day 1 to 3). Similarly, it was assumed that birds that were infected at the day of logger removal (e.g. day 20) had also been infected the three days prior to removal of the logger (e.g. day 17 to 19). These assumptions are based on the fact that in free-living mallards the length of LPAIV shedding is between three and eight days (LatorreMargalef et al. 2009). Non-infected individuals at the day of logger deployment as well as logger removal were assumed to stay uninfected three days after logger deployment and prior to logger removal. Using other tracking periods (e.g. day 1 after logger deployment, day 3 after logger deployment, last day prior to logger removal, the full tracking period) did not yield other results, showing the robustness of this three-day period.

The tracking data for the first three days after logger deployment were included in the analysis for 71 of the 74 mallards fitted with GPS loggers and sampled for LPAIV. We excluded three birds from the analysis: one individual which had only two tracking days recorded, and two birds for which the time spent away from the roost could not be calculated properly (no consecutive positions indicating that the individual was $>50 \mathrm{~m}$ from the roost). We only had tracking data of the three days before logger removal of 22 of the 74 mallards, since most individuals were recaptured and sampled for LPAIV after 18 days when the batteries of the GPS logger had run out of power.

The five movement parameters, body mass and the weather parameters were $\log 10$-transformed to meet the assumption of normality. Collinearity between the five movement parameters and the three weather parameters was tested using Pearson correlation (r). Daily number of flights, daily flight distance, daily home range and daily time away from the roost were highly correlated (Supplementary material Appendix 3 Table A1). There were no correlations between daily local movements and the other movement parameters, except home range $(r=0.15)$. However due to the low $\mathrm{r}^{2}(0.02)$, daily local movements were retained in the models to test the variance in movement patterns in relation to LPAIV infection status. A principal component (PC) analysis was performed on the four highly correlated movement parameters to create a first PC (PC1) to use as an index of daily regional movements (PC1 explained 66\% of the variance, PC2 only $16 \%$, eigenvalue $=2.644$ ). For the weather parameters, collinearity existed between wind speed and precipitation (Supplementary material Appendix 3 Table A2). A PC analysis was performed on wind speed and precipitation, and the $\mathrm{PC} 1$ was used as index for windprecipitation (PC1 explained $81 \%$ of the variance, PC2 only $19 \%$, eigenvalue $=1.619$ ). As an index of bird body size the $\mathrm{PC} 1$ of a PC analysis of tarsus, head + bill and wing lengths is commonly used. The PC1 of body size explained $49 \%$ of the variance $(\mathrm{PC} 2$ only $30 \%$, eigenvalue $=1.467)$. Correlations between the $\mathrm{PC} 1 s$ and parameters (i.e. factor loadings) are shown in Table A3 (Supplementary material Appendix 3).

For the analysis within individuals, we selected only those individuals of which both tracking data of the first three days after GPS logger deployment (day 1-3) and the last three days before logger removal was known $(n=22$; for details see Supplementary material Appendix 2 Fig. A2). We used linear mixed models (LMMs) to test the associations between LPAIV infection and daily local and regional movements within individuals, with LPAIV infection status as fixed factor, and individual bird as random factor to correct for repeated measures. As covariates we included tracking day (i.e. day 1, 2 and 3 of the three-day period), tracking period (i.e. three days following GPS deployment or three days prior to logger removal), wind-precipitation and temperature. Furthermore, we included the interactions between infection status and wind-precipitation, and infection status and temperature to test whether movements of LPAIV infected and non-infected individuals were influenced by weather conditions.

For the analysis among individuals, infected or not, we used tracking data recorded 1) in the first three days after capture $(\mathrm{n}=71)$ and 2$)$ in the last three days before logger removal $(\mathrm{n}=22$; for details see Supplementary material Appendix 2 Fig. A3-A4). We analysed both tracking periods separately to include additional covariates (antibody status, body mass, bird size) in the models using the tracking data of the first three days after logger deployment. Moreover, bird movements after logger deployment might be affected by handling. We used LMMs to test associations between LPAIV infection and daily local and regional movements between birds. The LMMs included LPAIV infection status as fixed factor, and tracking day, wind-precipitation and temperature as covariates, and two-way interactions between infection status and wind-precipitation, and infection status and temperature, and individual bird as random factor. The LMM for the first three days also included antibody status, body mass and bird size as covariates, and the interaction between infection status and antibody status, and infection status and body mass. Where interactions were significant, these were explored further by modelling infected and noninfected birds separately. Six out of 10 of the LPAIV infected mallards at logger deployment had AIV antibodies. AIV antibodies in infected mallards reflect previous infection, whereas infected birds without AIV antibodies probably have no (recent) infection history. Since there might exist a potential difference in daily movements between birds with previous infections and individuals that were immunologically naïve, antibody status was included as a covariate. AIV antibodies were not correlated with LPAIV infection $\left(\chi_{69}^{2}=0.200, p=0.655\right)$. In order to correct for differences in body condition among individuals, body mass, and bird size, to adjust body mass for structural size, were included in the model. The interaction between infection status and body mass was included, since body mass may differ between LPAIV infected and non-infected birds (LatorreMargalef et al. 2009). Antibody status and body mass were not included in the LMMs using tracking data recorded in the last three days, since these measurements were collected at the day of GPS logger deployment and cannot be extrapolated to the last days.

Bird's origin was not included in the LMMs. This study was conducted in the period that both resident and migratory mallards were present at our study site. Based on a study by van Dijk et al. (2014), who assessed the origin of mallards captured at our study site in autumn 2010 using re-sighting data and hydrogen stable isotope analysis in feathers, we could determine the origin of 17 mallards: 7 residents and 10 migrants. Using the first three days after GPS logger deployment, local and regional movements did 
not differ between resident and migratory mallards (respectively, LMM: $t_{1,49}=0.410, p=0.680$ and $t_{1,49}=-1.733$, $\mathrm{p}=0.090)$.

All analyses were conducted using $\mathrm{R}$ ver. 2.14.1 $(<$ www.r-project.org/ $>$ ). Package lme4 was used to fit LMMs (Bates et al. 2012), and package languageR to generate output of LMMs and upper and lower $95 \%$ confidence intervals (Baayen 2011). The latter was used to assess effect sizes of non-significant results: a narrow breadth is more consistent with the null hypothesis of no effect, whereas large confidence intervals may indicate low statistical power (Colegrave and Ruxton 2003).

\section{Results}

At the day of GPS logger deployment, 14\% (10 out of 71) of mallards were infected with LPAIV and 66\% (47 out of 71) had antibodies against AIV. At the day of logger removal, $59 \%$ (13 out of 22) of mallards were infected with LPAIV, with $9 \%$ (2 out of 22) of these individuals infected at both logger deployment and removal. Whereas 9\% (2 out of 22) of these mallards were infected only at logger deployment (non-infected at logger removal) and 50\% (11 out of 22) only at logger removal (non-infected at logger deployment).

\section{Associations between infection and daily movements within individuals}

Within individuals, there was no association between LPAIV infection and regional movements (Table 1). There was a significant interaction effect between LPAIV infection status and wind-precipitation on local movements (Table 1). When uninfected, local movements were not associated with wind-precipitation $\left(t_{1,77}=0.680, p=0.522, C I=-0.016\right.$ $0.032)$, but when infected with LPAIV their local movements tended to decrease with an increase in wind-precipitation $\left(\mathrm{t}_{1,47}=-1.990, \mathrm{p}=0.058, \mathrm{CI}=-0.086-0.002\right)$. Neither local nor regional movements within individuals were associated with temperature (Table 1).

\section{Associations between infection and daily movements among individuals}

Among individuals, local movements were not associated with LPAIV infection (Table 2a, Fig. 2a). Regional movements recorded during the first days of tracking were also not associated with LPAIV infection (Table 2b). However, regional movements recorded over the last days of tracking were lower in LPAIV infected than in non-infected mallards (Table 2b, Fig. 2b). Ignoring the other variables in the statistical model, in order to get a notion of the potential effect of LPAIV infection on daily regional movements, the mean \pm SE of each regional movement parameter for LPAIV infected and non-infected mallards were, respectively: number of flights: $3.4 \mathrm{~d}^{-1}( \pm 0.3)$ versus $3.9 \mathrm{~d}^{-1}( \pm 0.4)$, flight distance: $1314 \mathrm{~m} \mathrm{~d}^{-1}( \pm 162)$ versus $1842 \mathrm{~m} \mathrm{~d}^{-1}$ ( \pm 209$)$, home range: $0.16 \mathrm{~km}^{2} \mathrm{~d}^{-1}( \pm 0.02)$ versus $0.20 \mathrm{~km}^{2} \mathrm{~d}^{-1}$ ( \pm 0.03$)$, time away from the roost: $430 \mathrm{~min} \mathrm{~d}^{-1}( \pm 45)$ versus $602 \mathrm{~min} \mathrm{~d}^{-1}$ ( \pm 44) (Supplementary material Appendix 2 Fig. A4).

There was an interaction effect between LPAIV infection status and temperature for local movements recorded in the first days of tracking (Table 2a). Local movements of LPAIV infected birds were higher when temperatures were lower $\quad\left(t_{1,24}=-2.437, \quad p=0.040, \quad C I=-1.682--0.064\right.$; Fig. 3a), while there was no association between local movements of non-infected birds and temperature $\left(\mathrm{t}_{1,177}=-0.328, \quad \mathrm{p}=0.762, \quad \mathrm{CI}=-0.044-0.059 ; \quad\right.$ Fig. $3 \mathrm{~b})$. Also between LPAIV infection status and both windprecipitation and temperature there was an interaction effect for regional movements recorded in the last days of tracking (Table 2b). LPAIV infected birds were flying less and shorter distances, had smaller home ranges and spent less time away from the roost, when there was more wind and precipitation $\left(t_{1,36}=-1.696, p=0.022, C I=-2.792--0.270\right.$; Fig. 4a $)$, and temperatures were lower $\left(\mathrm{t}_{1,36}=4.137, \mathrm{p}<0.001, \mathrm{CI}=5.035-13.555\right.$; Fig. 4b). Regional movements of non-infected birds were not associated with wind-precipitation $\left(\mathrm{t}_{1,24}=1.245, \mathrm{p}=0.292\right.$, $\mathrm{CI}=-0.427-1.495$; Fig. $4 \mathrm{c})$ and temperature $\left(\mathrm{t}_{1,24}=-0.523\right.$, $\mathrm{p}=0.676, \mathrm{CI}=-4.746-3.219$; Fig. $4 \mathrm{~d})$.

There were no correlations between weather and local movements of individuals (Table 2a). However, regional movements recorded over the first days of tracking were associated with temperature (Table 2b): mallards were flying less and shorter distances, had smaller home ranges and spent less time away from the roost when temperatures were lower. Antibody status and body mass were not associated with local and regional movements of birds (Table 2).

\section{Discussion}

Daily regional movements $(>100 \mathrm{~m})$ of mallards in the last days of tracking were smaller in LPAIV infected individuals than non-infected individuals. Based on uncorrected values, LPAIV infected mallards were flying at least 10\%

Table 1. 95\% confidence intervals and test statistics for parameter estimates of two linear mixed models testing the effects of LPAIV infection and a range of weather and tracking conditions (see text) on daily local and daily regional movements within individuals $(n=22, D F=125)$. Significant p-values are in bold.

\begin{tabular}{llrrrrrr}
\hline & \multicolumn{3}{c}{ Local movements } & & \multicolumn{3}{c}{ Regional movements } \\
\cline { 2 - 3 } Variable & $95 \% \mathrm{Cl}$ & $\mathrm{t}$-value & $\mathrm{p}$-value & & $95 \% \mathrm{Cl}$ & $\mathrm{t}$-value & $\mathrm{p}$-value \\
\hline Infection status & $-0.086-0.064$ & -0.210 & 0.832 & & $-1.222-1.413$ & 0.115 \\
Tracking day & $-0.017-0.015$ & -0.260 & 0.797 & & $-0.280-0.257$ & -0.086 & 0.909 \\
Tracking period & $-0.230-0.286$ & 0.380 & 0.703 & & $-3.891-4.841$ & 0.214 & 0.831 \\
Wind-precipitation & $-0.024-0.031$ & 0.280 & 0.778 & & $-0.502-0.492$ & -0.245 & 0.807 \\
Temperature & $-0.086-0.068$ & -0.160 & 0.876 & & $-2.283-0.424$ & -1.326 & 0.187 \\
Infection status $\times$ Wind-precipitation & $-0.089--0.003$ & -2.150 & $\mathbf{0 . 0 3 3}$ & & $-1.291-0.290$ & -1.149 & 0.253 \\
Infection status $\times$ Temperature & $-0.042-0.183$ & 1.210 & 0.229 & & $-1.550-2.434$ & 0.520 & 0.604 \\
\hline
\end{tabular}


Table 2. 95\% confidence interval and test statistics for parameter estimates of four linear mixed models testing the effects of LPAIV infection and a range of weather factors, tracking conditions and bird characteristics (see text) on (a) daily local and (b) daily regional movements among individuals. Different tests were performed for the first three days after GPS logger deployment $(n=71, D F=202)$ and the last three days prior to logger removal $(n=22, D F=60)$. Significant $p$-values are in bold.

\begin{tabular}{|c|c|c|c|c|c|c|}
\hline \multirow[b]{2}{*}{ Variable } & \multicolumn{3}{|c|}{ First three days } & \multicolumn{3}{|c|}{ Last three days } \\
\hline & $95 \% \mathrm{Cl}$ & t-value & p-value & $95 \% \mathrm{Cl}$ & t-value & p-value \\
\hline Infection status & $0.538-10.084$ & 1.612 & 0.109 & $-0.067-0.433$ & 1.613 & 0.112 \\
\hline Tracking day & $-0.004-0.024$ & 1.755 & 0.081 & $-0.034-0.012$ & -0.998 & 0.323 \\
\hline Antibody & $-0.023-0.039$ & 0.437 & 0.663 & & & \\
\hline Body mass & $-0.357-0.482$ & -0.016 & 0.987 & & & \\
\hline Bird size & $-0.003-0.025$ & 1.376 & 0.170 & & & \\
\hline Wind-precipitation & $-0.005-0.005$ & 0.742 & 0.459 & $-0.042-0.053$ & 0.187 & 0.852 \\
\hline Temperature & $-0.048-0.065$ & -0.229 & 0.819 & $-0.190-0.216$ & 0.166 & 0.869 \\
\hline Infection status $\times$ Antibody & $-0.106-0.069$ & -0.377 & 0.707 & & & \\
\hline Infection status $\times$ Body mass & $-2.871-0.055$ & -1.378 & 0.170 & & & \\
\hline Infection status $\times$ Wind-precipitation & $-0.059-0.031$ & -0.724 & 0.470 & $-0.075-0.063$ & -0.135 & 0.893 \\
\hline Infection status $\times$ Temperature & $-1.412--0.305$ & -2.727 & 0.007 & $-0.430-0.084$ & -1.443 & 0.154 \\
\hline \multicolumn{7}{|l|}{ (B) } \\
\hline & \multicolumn{6}{|c|}{ Regional movements } \\
\hline & \multicolumn{3}{|c|}{ First three days } & \multicolumn{3}{|c|}{ Last three days } \\
\hline Variable & $95 \% \mathrm{Cl}$ & t-value & p-value & $95 \% \mathrm{Cl}$ & t-value & p-value \\
\hline Infection status & $-53.323-87.016$ & 0.423 & 0.673 & $-15.728--6.141$ & -4.297 & $<0.001$ \\
\hline Tracking day & $-0.233-0.184$ & 0.009 & 0.993 & $-0.127-0.747$ & 0.508 & 0.613 \\
\hline Antibody & $-0.407-0.501$ & 0.182 & 0.856 & & & \\
\hline Body mass & $-3.197-9.125$ & 0.586 & 0.559 & & & \\
\hline Bird size & $-0.097-0.312$ & 0.967 & 0.335 & & & \\
\hline Wind-precipitation & $-0.097-0.217$ & -0.419 & 0.675 & $-0.410-1.408$ & 1.642 & 0.106 \\
\hline Temperature & $1.506-3.126$ & 4.390 & $<0.001$ & $-4.795-2.918$ & -0.731 & 0.468 \\
\hline Infection status $\times$ Antibody & $-1.961-0.602$ & -0.793 & 0.429 & & & \\
\hline Infection status $\times$ Body mass & $-25.691-17.132$ & -0.408 & 0.684 & & & \\
\hline Infection status $\times$ Wind-precipitation & $-0.326-1.043$ & 1.522 & 0.130 & $-3.317--0.721$ & -2.941 & 0.005 \\
\hline Infection status $\times$ Temperature & $-9.697-6.841$ & -0.232 & 0.817 & 5.292-15.169 & 3.952 & $<0.001$ \\
\hline
\end{tabular}

less, home ranges were $20 \%$ smaller, and flight distance and time away from the roost (i.e. duck decoy) were both almost $30 \%$ lower. Placing our findings in the context of regional spread of LPAIV by mallards, this would suggest that LPAIV infected mallards would cover between 16 and $43 \mathrm{~km}$ less in an infectious period of three to eight days than non-infected mallards. Non-infected mallards at our study site could cover a maximum distance of $18 \mathrm{~km}$ a day. During the peak of
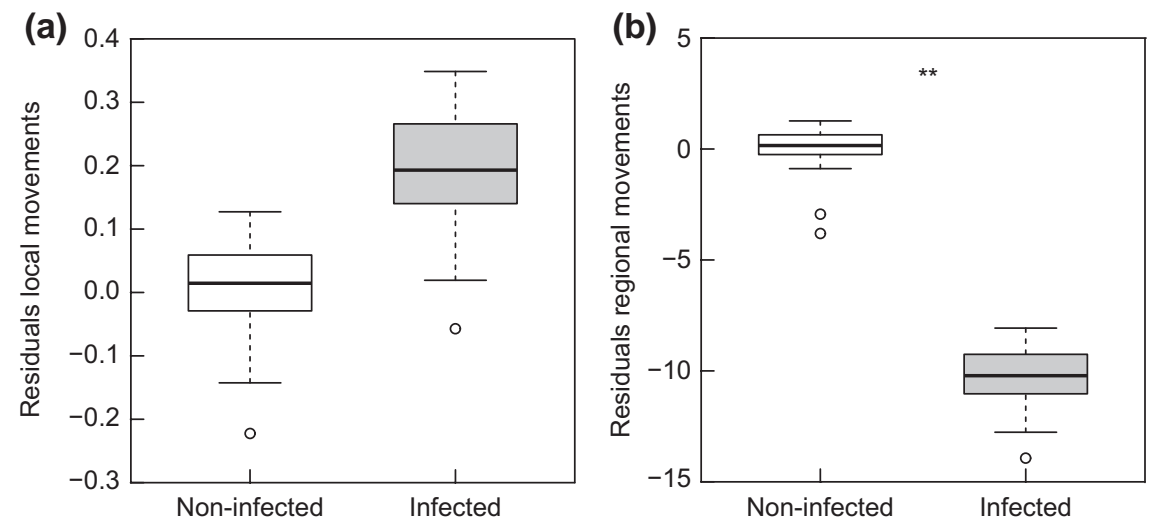

Figure 2. LPAIV infection status among individuals $(\mathrm{n}=22)$, associated with (a) daily local movements $(<100 \mathrm{~m})$ and $(\mathrm{b})$ daily regional movements $(>100 \mathrm{~m})$ in the last three days prior to logger removal. The y-axis shows the partial residuals of the local and regional movements in which the other fixed factors used in the linear mixed models are also included. Regional movements are positively correlated with daily number of flights, daily flight distance, daily home range and daily time away from the roost (Supplementary material Appendix 3 Table A3). Box plots represent medians and 25\%- and 75\% quartiles, with whiskers signifying the range of the data excluding extreme outliers (dots). ${ }^{* *}$ indicates $\mathrm{p}<0.05$. 
(a)

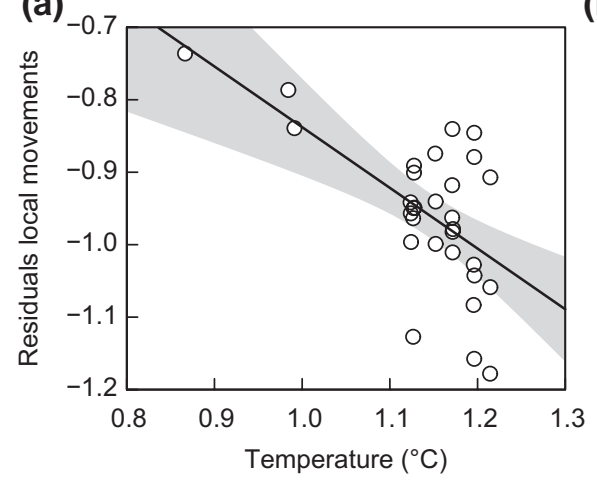

(b)

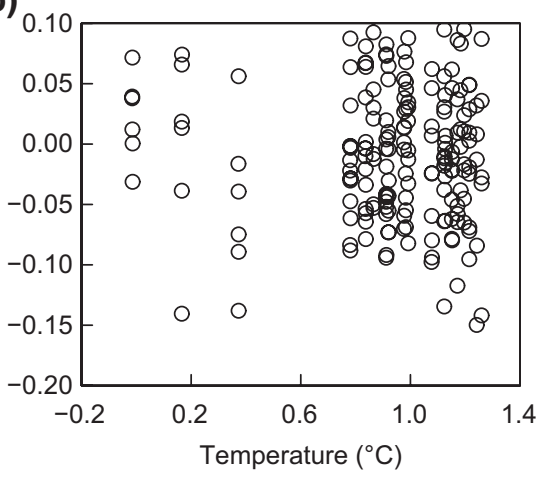

Figure 3. Daily local movements $(<100 \mathrm{~m})$ recorded during the first three days of tracking after GPS logger deployment between (a) LPAIV infected ( $\pm 95 \% \mathrm{CI}$ ) and (b) non-infected mallards and their relationship with temperature (note log-scale; $\mathrm{n}=71$ ). The $\mathrm{y}$-axes show the partial residuals of the local movements in which the other fixed factors used in the linear mixed model are also included.

infection in autumn, mallards are likely to become infected more than once. Therefore the total regional movements of each individual in autumn could be much lower, hereby reducing the spread of LPAIV. In addition, the spread of LPAIV will likely also be restricted as a result of the typical regional movement routines of mallards in autumn, which are characterized by daily flights to the same nearby foraging areas (Kleyheeg unpubl.). Although LPAIV infected mallards are still able to spread the virus, LPAIV may indeed be spread over a smaller area than if infection does not hamper movement.

Our findings correspond with the hampered premigratory movements found in Bewick's swans infected with the same virus, although in that study movements were based on resightings of individuals (i.e. displacement; van Gils et al. 2007), and a follow-up study could not replicate these findings (Hoye 2011). Similar negative effects of pathogen infection on host movement using GPS loggers were found in other host-pathogen systems, such as Iberian ibex Capra pyrenaica affected by Sarcoptes scabiei (Alasaad et al. 2013) and bighorn sheep Ovis canadensis infected with keratoconjunctivitis (Jansen et al. 2007). Despite this, many studies make inferences about the consequences of animal movements for disease transmission without knowing whether pathogen infection affects those movements (Wyckoff et al. 2009, Woodroffe and Donnelly

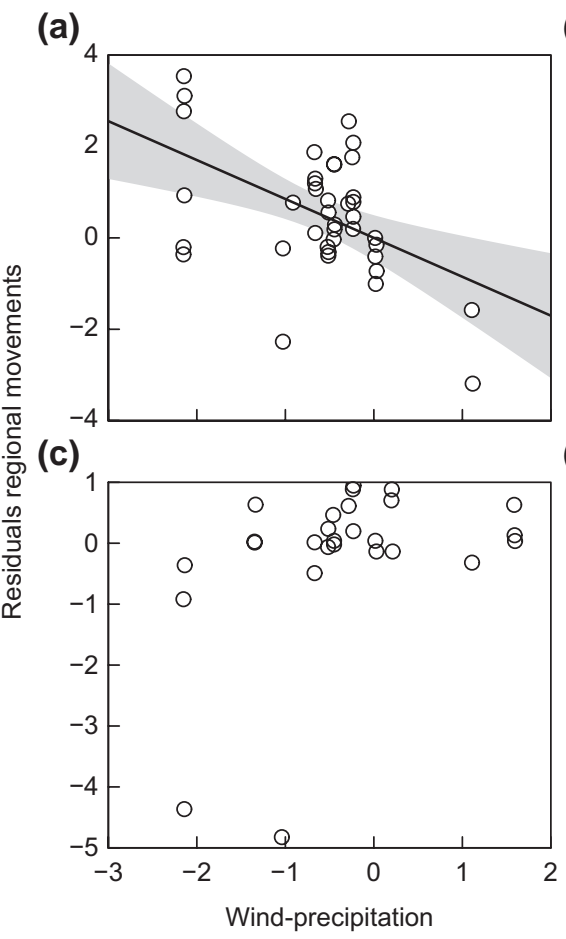

(b)

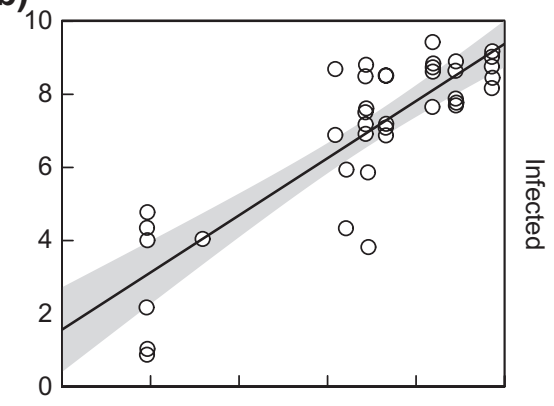

(d)

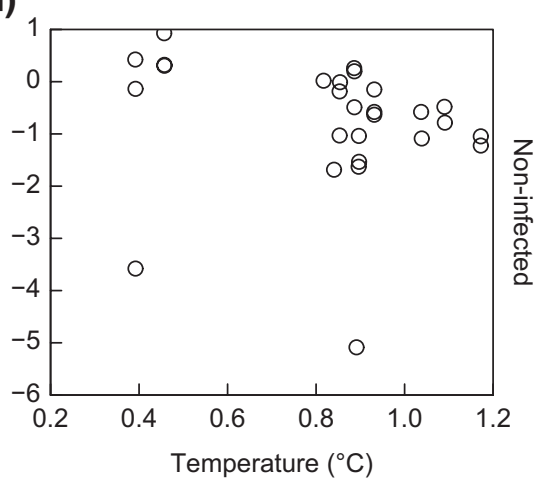

Figure 4. Daily regional movements $(>100 \mathrm{~m})$ recorded during the last three days of tracking prior to GPS logger removal between LPAIV infected and non-infected mallards $(\mathrm{n}=22)$ and their relationship with wind-precipitation ( $\mathrm{a}$ and $\mathrm{c}, \pm 95 \% \mathrm{CI})$ and temperature $(\mathrm{b}$ and $\mathrm{d}$; note log-scale). The y-axes show the partial residuals of the regional movements in which the other fixed factors used in the linear mixed model are also included. Regional movements are positively correlated with daily number of flights, daily flight distance, daily home range and daily time away from the roost. Wind-precipitation is positively correlated with wind speed and precipitation (Supplementary material Appendix 3 Table A3). 
2011). This is also frequently the case in studies predicting HPAIV H5N1 dispersal extrapolating from GPS-tracking studies in non-infected individuals (Gaidet et al. 2010, Newman et al. 2012). Even though LPAIV infection was likely associated with mallard movements in this study, infected individuals were probably still capable of transporting LPAIV to other areas: most LPAIV infected individuals still left the roosting site at night to fly to their foraging areas, making it plausible that they shed viral particles in the surface water when foraging, potentially infecting other birds.

Since this is a correlative study we cannot exclude the possibility that instead of LPAIV infection lowering mallards' regional movements, it is lower regional movements that increase a mallard's risk of LPAIV infection. This may occur when birds stay in a highly infected area (i.e. with many viral particles in the water). Although viral prevalence in mallards at our study site was similar to that of mallards at other locations in the Netherlands (45 to $135 \mathrm{~km}$ from the duck decoy; van Dijk 2014), we cannot exclude that individuals with lower regional movements became infected by the locally circulating LPAIV strains at our study site. Another possibility could be that mallards with lower regional movements were already less fit, and hence more susceptible for LPAIV infection. However, our study showed no significant interaction effect between infection and body mass.

In contrast to regional movements, daily local movements $(<100 \mathrm{~m})$ of mallards were not negatively associated with LPAIV infection. Local movements were assumed to involve no flights, whereas regional movements did. Hence, a potential explanation for why we found an association between LPAIV infection and daily regional movements but not local movements could be the higher energetic (flight) costs of regional compared to local movements. Nudds and Bryant (2000) showed that particularly short flights in birds, also involving take-off, landing, ascent and descent, are extremely costly (nearly 28 times basal metabolic rate in their study object, the zebra finch Taenuipygia guttata). Mounting and maintaining an immune response is believed to be energetically costly, requiring substantial protein and nutrient supplies (Lochmiller and Deerenberg 2000). It is therefore possible that LPAIV infected birds were less capable of investing this extra energy in regional movements due to the costs of clearing the infection.

Weather was associated with the daily regional movements of LPAIV infected mallards that were recorded before logger removal. LPAIV infected mallards during this period were flying less and shorter distances, had smaller home ranges and spent less time away from the roost when it was colder, windier and/or there was more rain. An opposite trend was apparent for daily local movements of LPAIV infected birds which increased with colder weather. Sauter et al. (2012) showed that travelling distance of mallards decreased with an increase in wind speed and precipitation, but in our study the correlation was only shown in LPAIV infected mallards. There was no association between both wind speed and precipitation and local and regional movements of non-infected birds. Normally, ducks reduce feeding activity with more wind and precipitation (Paulus 1988), but increase foraging time when temperatures decrease (Sauter et al. 2010). This is also shown by our results with LPAIV infected mallards reducing feeding activity such as flight (regional movements between roost and foraging areas) with more wind and rain, but increasing foraging time (local movements at foraging areas) when temperature decreases. It is possible that the harsher weather conditions reduced overall foraging and negatively affected body condition of LPAIV infected individuals. Latorre-Margalef et al. (2009) showed that LPAIV infected mallards tend to have slightly less body fat stores than non-infected birds.

Differences in daily regional movements between LPAIV infected and non-infected mallards were only detected using the tracks immediately prior to GPS logger removal, and not after logger deployment. This may have been caused by handling of the birds to fit the GPS loggers. We made an attempt to correct for such a handling effect by disregarding the GPS tracks recorded until noon on the day following GPS deployment. But it is possible that, this period was not long enough to account for the handling effect and for the birds to become accustomed to the logger; a study in captive mallards fitted with GPS loggers showed that effects of handling on mallard behaviour decreased significantly from day three onwards (Kleyheeg unpubl.). This handling effect could also be responsible for our failure to find differences in daily local and regional movements within individuals. Indeed, the movement differences we were able to detect between LPAIV infected and non-infected birds were subtle. Its detection may therefore have been best guaranteed long after the birds were equipped with a GPS logger and had become fully accustomed to wearing it. Another potential reason for not finding an association between LPAIV infection and mallard movements recorded in the first three days after logger deployment could be that all LPAIV-positive birds were positive in the oropharynx. It is currently debated whether wild birds that are tested positive for LPAIV in the oropharynx are infected, since the respiratory tract of these birds contains no detectible virus antigen (Wille et al. 2014). The significant results found in the analyses of the regional movements recorded over the last days before logger removal did contain LPAIV-positive mallards that were also positive in the cloaca (23\%: cloaca-positive, $31 \%$ : oropharynxpositive, $46 \%$ : cloaca- and oropharynx-positive). However, reanalysis of our data using only those mallards that were LPAIV-positive in the cloaca did not show other significant results or change our conclusions.

The confidence intervals of the parameter estimates in the within-individual comparisons for birds that were infected at one stage and non-infected at another were relatively small (Table 1) suggesting sufficient statistical power for these comparisons. Although we did find a significant association between LPAIV infection and regional movements recorded on the last three days prior to logger removal between individuals, the confidence intervals of the analyses using the first three days after logger deployment were (very) large (Table 2). The latter suggests a lack of statistical power and an insufficient sample size to make meaningful comparisons between infected and non-infected individuals directly after logger deployment.

Overall, we found weak negative associations between LPAIV infection and movement behaviour in mallards. Together with findings of other studies that showed weak negative associations between LPAIV infection and mallard's body condition and immune status (Latorre-Margalef et al. 
2009, van Dijk et al. 2015), this raises the question as to whether this is a consequence of a co-evolution between mallards and LPAIV. Hosts and pathogens may co-evolve (i.e. go through a process of reciprocal, adaptive genetic changes) if their relationship is close and strong selective pressures exist, acting on both the host and pathogen (Woolhouse et al. 2002). A co-evolution between mallards and LPAIV could both explain the observed weak effects of infection on mallard movements as well as their role as one of the key reservoirs for LPAIV.

In conclusion, by comparing movements of infected and non-infected individuals of a key LPAIV host species, we showed a weak negative association between LPAIV infection and daily regional movements of mallards. Particularly, LPAIV infected birds responded more strongly to adverse weather conditions, and flew less and over shorter distances, occupying a smaller home range when it was colder, more windy and/ or rainy. Differences in daily regional movements were only found between LPAIV infected and non-infected individuals and could not be detected within individuals. There was no association between LPAIV infection and daily local movements, although local movements of LPAIV infected mallards were higher when temperatures were lower. Our study suggests that LPAIV infection impairs bird movements, albeit to a limited extent only, suggesting potential for dispersal of LPAIV by infected birds. With this study we contribute to assessing the potential role birds play in the spread of LPAIV and more generally, highlight the critical importance of pathogen infection on animal movements in assessing a host's potential role in the emergence and spread of a pathogen.

Acknowledgements - J. G. B. van Dijk and E. Kleyheeg contributed equally to this work. We express our gratitude to Teunis de Vaal, who captured all mallards and assisted with sampling the birds and fitting them with GPS loggers. Kees Oosterbeek provided critical advice on the usage of teflon for harness construction. We thank Peter de Vries, Tara Woud and Dieuwertje Boonstra for assistance in the field, and Oanh Vuong and Judith Guldemeester for laboratory assistance. Finally, we thank Sjoerd Duijns and Alice Risely for comments on earlier versions of this paper. This study was supported by the Netherlands Organization for Scientific Research (NWO-ALW; Vidi grant 864.10.006 to MBS and grant 820.01 .018 to MK), the National Institute of Health (NIH; contract NIAID HHSN266200700010C), the Royal Netherlands Institute for Sea Research (NIOZ), Texel (supporting JvD), and the Australian Research Council. Mallards were handled under approval from the Animal Experiment Committee (protocol UU2012.I.01.009). All efforts were made to minimize animal discomfort throughout the study. This is publication 5765 of the NIOO-KNAW.

\section{References}

Alasaad, S. et al. 2013. The use of radio-collars for monitoring wildlife diseases: a case study from Iberian ibex affected by Sarcoptes scabiei in Sierra Nevada, Spain. - Parasites Vectors 6: $1-5$.

Alexander, D. J. 2007. An overview of the epidemiology of avian influenza. - Vaccine 25: 5637-5644.

Altizer, S. et al. 2011. Animal migration and infectious disease risk. - Science 331: 296-302.
Baayen, R. H. 2011. LanguageR: Data sets and functions with "Analyzing Linguistic Data: A practical introduction to statistics". - R package ver. 1.4. < http://CRAN.R-project.org/package $=$ language $\mathrm{R}>$.

Bates, D. et al. 2012. Lme4: linear mixed-effects models using S4 classes. - R package ver. 0.999999-0. < http://CRAN.R-project. org $/$ package $=\operatorname{lme} 4>$.

Bradley, C. A. and Altizer, S. 2005. Parasites hinder monarch butterfly flight: implications for disease spread in migratory hosts. - Ecol. Lett. 8: 290-300.

Calenge, C. 2006. The package adehabitat for the R software: a tool for the analysis of space and habitat use by animals. - Ecol. Modell. 197: 516-519.

Colegrave, N. and Ruxton, G. D. 2003. Confidence intervals are a more useful complement to nonsignificant tests than are power calculations. - Behav. Ecol. 14: 446-450.

Cramp, S. and Simmons, K. E. L. 1977. Handbook of the Birds of Europe, the Middle East and North Africa: the birds of the western Palearctic, Vol. 1: Ostrich to Ducks. - Oxford Univ. Press.

Delahay, R. J. et al. 1995. The energetic consequences of parasitism: effects of a developing infection of Trichostrongylus tenuis (Nematoda) on red grouse (Lagopus lagopus scoticus) energy balance, body weight and condition. - Parasitology 110: 473-482.

Delany, S. and Scott, D. 2006. Waterbird population estimates, 4th edn. - Wetlands Int.

Fellous, S. et al. 2011. Parasitic infection reduces dispersal of ciliate host. - Biol. Lett. 7: 327-329.

Gaidet, N. et al. 2010. Potential spread of highly pathogenic avian influenza $\mathrm{H} 5 \mathrm{~N} 1$ by wildfowl: dispersal ranges and rates determined from large-scale satellite telemetry. - J. Appl. Ecol. 47: 1147-1157.

Hesterberg, U. et al. 2009. Avian influenza surveillance in wild birds in the European Union in 2006. - Influenza Other Respir. Viruses 3: 1-14.

Hoye, B. J. 2011. Host-pathogen interactions on the move: migratory waterfowl and avian influenza viruses. - PhD thesis, Utrecht Univ.

Hoye, B. J. 2012. Variation in postsampling treatment of avian blood affects ecophysiological interpretations. - Meth. Ecol. Evol. 3: 162-167.

Jansen, B. D. et al. 2007. Population dynamics and behavior of bighorn sheep with infectious keratoconjunctivitis. - J. Wildl. Manage. 71: 571-575.

Kida, H. et al. 1980. Duck influenza lacking evidence of disease signs and immune response. - Infect. Immun. 30: 547-553.

Kilpatrick, A. M. et al. 2006. Predicting the global spread of H5N1 avian influenza. - Proc. Natl Acad. Sci. USA 103: 19368-19373.

Kim, Y.-I. et al. 2014. Pathobiological features of a novel, highly pathogenic avian influenza A(H5N8) virus. - Emerg. Microbes Infect. 3: e75.

Kleijn, D. et al. 2010. Dynamics and ecological consequences of avian influenza virus infection in greater white-fronted geese in their winter staging areas. - Proc. R. Soc. B 277: 2041-2048.

Kreijtz, J. H. C. M. et al. 2013. Low pathogenic avian influenza $\mathrm{A}(\mathrm{H} 7 \mathrm{~N} 9)$ virus causes high mortality in ferrets upon intratracheal challenge: a model to study intervention strategies. - Vaccine 31: 4995-4999.

Kuiken, T. 2013. Is low pathogenic avian influenza virus virulent for wild waterbirds? - Proc. R. Soc. B 280: 20130990.

Latorre-Margalef, N. et al. 2009. Effects of influenza A virus infection on migrating mallard ducks. - Proc. R. Soc. B 276: 1029-1036.

Latorre-Margalef, N. et al. 2014. Long-term variation in influenza A virus prevalence and subtype diversity in migratory mallards in northern Europe. - Proc. R. Soc. B 281: 20140098. 
Lee, Y.-J. et al. 2014. Novel reassortant influenza A(H5N8) viruses, South Korea, 2014. - Emerg. Infect. Dis. 20: 1086-1089.

Lochmiller, R. L. and Deerenberg, C. 2000. Tradeoffs in evolutionary immunology: just what is the cost of immunity? - Oikos 88: 87-98.

Munster, V. J. et al. 2007. Spatial, temporal, and species variation in prevalence of influenza A viruses in wild migratory birds. - PLoS Pathog. 3: e61.

Newman, S. H. et al. 2012. Eco-virological approach for assessing the role of wild birds in the spread of avian influenza H5N1 along the Central Asian Flyway. - PLoS ONE 7: e30636.

Nudds, R. L. and Bryant, D. M. 2000. The energetic cost of short flights in birds. - J. Exp. Biol. 203: 1561-1572.

Olsen, B. et al. 2006. Global patterns of influenza A virus in wild birds. - Science 312: 384-388.

Paulus, S. L. 1988. Time-activity budgets of nonbreeding Anatidae: a review. - In: Wennerberg, L. (ed.), Waterfowl in winter. Univ. of Minnesota, pp. 135-152.

Prosser, D. J. et al. 2009. Satellite-marked waterfowl reveal migratory connection between $\mathrm{H} 5 \mathrm{~N} 1$ outbreak areas in China and Mongolia. - Ibis 151: 568-576.

Roshier, D. A. and Asmus, M. W. 2009. Use of satellite telemetry on small-bodied waterfowl in Australia. - Mar. Freshwater Res. 60: 299-305.

Sauter, A. et al. 2010. Evidence of climate chang effects on withinwinter movements of European mallards Anas platyrhynchos. - Ibis 152: 600-609.

Sauter, A. et al. 2012. Individual behavioural variability of an ecological generalist: activity patterns and local movements of mallards Anas platyrhynchos in winter. - J. Ornithol. 153: 713-726.

Scott, D. A. and Rose, P. M. 1996. Atlas of Anatidae populations in Africa and western Eurasia, Wetlands Int. Publ. No. 41. - Wetlands Int.

Takekawa, J. Y. et al. 2013. Movements of wild ruddy shelducks in the central Asian flyway and their spatial relationship to outbreaks of highly pathogenic avian influenza $\mathrm{H} 5 \mathrm{~N} 1$. - Viruses-Basel 5: 2129-2152.

van Dijk, J. G. B. 2014. Pathogen dynamics in a partial migrant: interactions between mallards (Anas platyrhynchos) and avian influenza viruses. - PhD thesis, Utrecht Univ.

Supplementary material (available online as Appendix oik.01836 at $<$ www.oikosjournal.org/readers/appendix $>$ ). Appendix 1-3. van Dijk, J. G. B. et al. 2014. Juveniles and migrants as drivers for seasonal epizootics of avian influenza virus. - J. Anim. Ecol. 83: 266-275.

van Dijk, J. G. B. et al. 2015. Minor differences in body condition and immune status between avian influenza virus-infected and noninfected mallards: a sign of coevolution? - Ecol. Evol. 5: 436-449.

van Gils, J. A. et al. 2007. Hampered foraging and migratory performance in swans infected with low pathogenic avian influenza A virus. - PLoS One 2: e184.

van Winkle, W. 1975. Comparison of several probabilistic home-range models. - J. Wildl. Manage. 39: 118-123.

Webster, R. G. et al. 1992. Evolution and ecology of influenza A viruses. - Microbiol. Rev. 56: 152-179.

Wille, M. et al. 2014. Infected or not: are PCR-positive oropharyngeal swabs indicative of low pathogenic influenza A virus infection in the respiratory tract of Mallard Anas platyrhynchos? - Vet. Res. 45: 53.

Woodroffe, R. and Donnelly, C. A. 2011. Risk of contact between endangered African wild dogs Lycaon pictus and domestic dogs: opportunities for pathogen transmission. - J. Appl. Ecol. 48: 1345-1354.

Woolhouse, M. E. J. et al. 2002. Biological and biomedical implications of the co-evolution of pathogens and their hosts. - Nat. Genet. 32: 569-577.

World Health Organization 2014a. WHO risk assessment of human infection with avian influenza $\mathrm{A}(\mathrm{H} 7 \mathrm{~N} 9)$ virus. <www.who.int/ influenza/human_animal_interface/influenza_h7n9/140225_ H7N9RA_for_web_20140306FM.pdf?ua=1> 28 February 2014.

World Health Organization 2014b. Cumulative number of confirmed human cases for avian influenza $\mathrm{A}(\mathrm{H} 5 \mathrm{~N} 1)$ reported to WHO, 2003-2014.<www.who.int/influenza/human_animal_ interface /EN_GIP_20140124CumulativeNumberH5N1cases. pdf?ua $=1 . p d f>24$ January 2014.

Wyckoff, A. C. et al. 2009. Feral swine contact with domestic swine: a serologic survey and assessment of potential for disease transmission. - J. Wildl. Dis. 45: 422-429.

Yorinks, N. and Atkinson, C. T. 2000. Effects of malaria on activity budgets of experimentally infected juvenile apapane (Himatione sanguinea). - Auk 117: 731-738. 